\title{
Nasolabial Cyst: Report of 2 Cases and Literature Review
}

\author{
Rodrigo Cabezón ${ }^{1 *}$ and Matías Willson ${ }^{2}$ \\ ${ }^{1}$ Department of Otolaryngology, Clínica Las Condes, Santiago, Chile \\ ${ }^{2}$ Department of Otolaryngology, Pontificia Universidad Católica de Chile, Santiago, Chile
}

*Corresponding author: Rodrigo Cabezón, Department of Otolaryngology, Clínica Las Condes, Santiago, Chile

\begin{abstract}
Introduction: Nasolabial cysts are a rare, ectodermal development cyst, presenting as a fullness of canine fossa, nasal ala or vestibule of the nose. They are usually asymptomatic but may become infected. Treatment is complete surgical excision by sublabial approach, or transanal endoscopic marsupialization.

Case Report: Description of two cases, one female presenting as nasal deformity due to progressive growth of unilateral nasolabial cyst, and a healthy young male presenting severe facial cellulitis, with a CT showing bilateral nasolabial cysts.

Discussion: Nasolabial cyst is a rare condition, but diagnosis and treatment are simple. Nasolabial cyst should be incorporated by ENT in the differential diagnosis of nose deformities and facial swelling.
\end{abstract}

\section{Introduction}

Nasolabial cysts are also described as nasoalveolar cyst or Klestadt cyst and were first described by Zuckerkandl [1,2]. They are rare, affecting 1,6 per 100.000 persons per year, more frequently in females (4:1 ratio), especially among African Americans, in the fourth and fifth decades of life. $90 \%$ are unilateral, and often underdiagnosed $[3,4]$. Nasolabial cysts are non-odontogenic cysts that develop lateral to the midline of the maxillary lip and alar base. They usually present as a swelling in the nasolabial fold, causing alar nose elevation and upper lip projection. They may grow slowly and painlessly over several years. Because of its close anatomical relation to the nasal cavity and teeth, they may become infected easily, rapidly growing and being painful $[1,5]$. Diagnosis is made by clinical examination, imaging tests, and is confirmed by histopathologic study. The cyst can be palpated bimanually with one finger in the floor of the nasal vestibule and another in the labial sulcus. Computed tomography (CT) and magnetic resonance (MR) can be useful. Differential diagnosis includes cysts of the nasopalatine duct, periapical inflammatory lesions (granuloma cyst, abscess), and epidermoid cysts [1,6]. Complete surgical excision of the nasolabial cyst is the best treatment, and sublabial approach is most used. Other authors propose transanal marsupialization as an easier and shorter procedure, with lesser complications, but recurrence may be a problem $[3,5]$.

\section{Case Report 1}

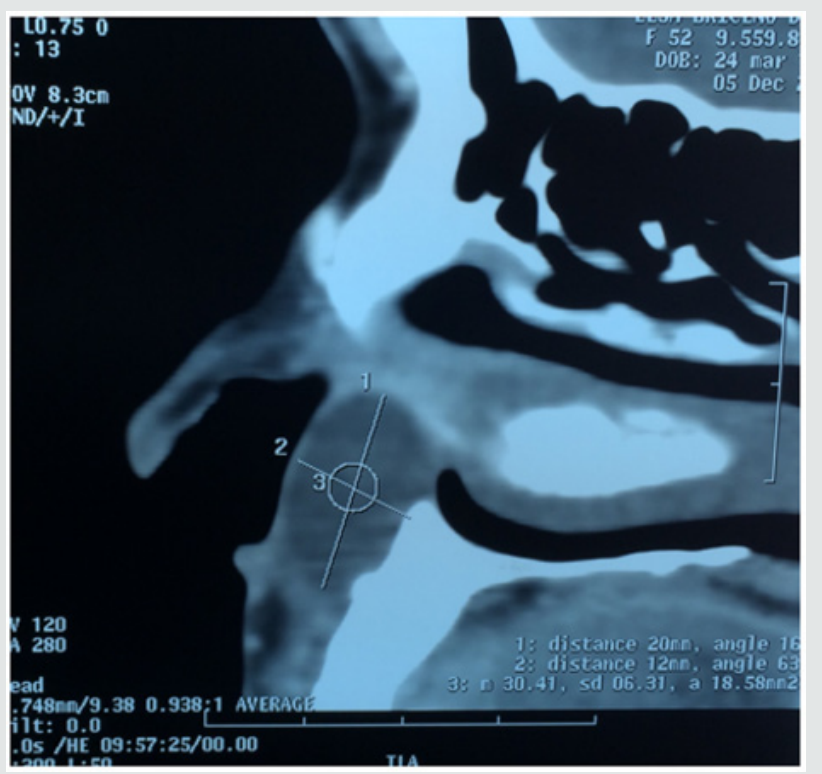

Figure 1(a).

52-year-old female patient with a 3-year history of left floor of nose intermittent painful swelling, and progressive deformity of this 
area. Computed tomography (CT) study showed a 3,2 $\mathrm{cm}$ rounded lesion in left pyriform aperture compatible with nasoalveolar cyst (Figure 1). A sublabial approach was performed, and complete excision of the cyst was obtained (Figure 2). Histological study confirmed suspected diagnosis. Patient evolved in excellent conditions, with no complications or recurrence in a 2 year follow up period.

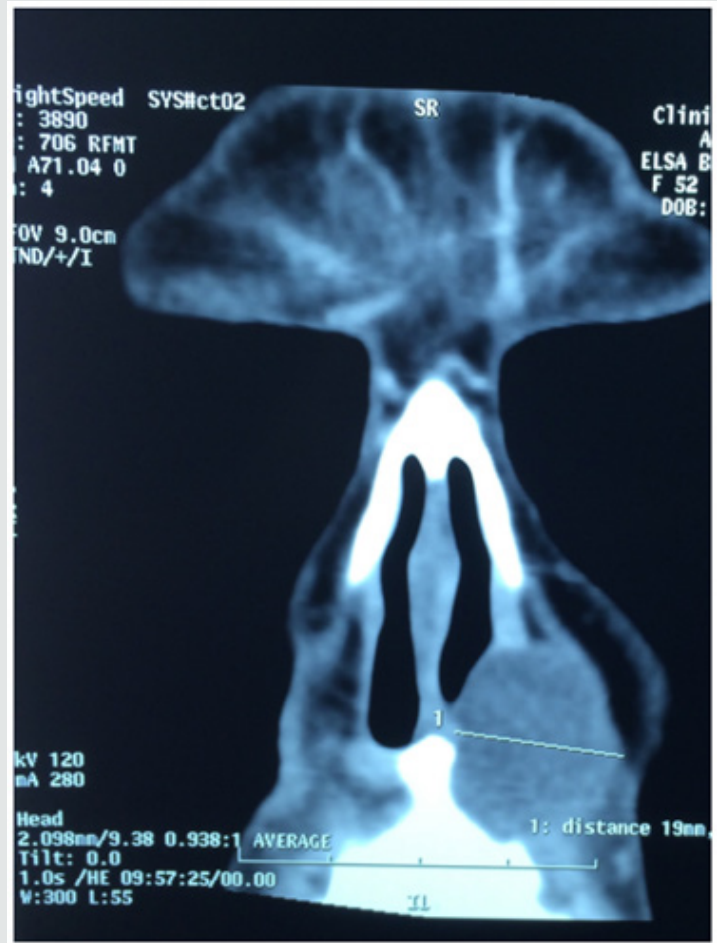

Figure 1(b).

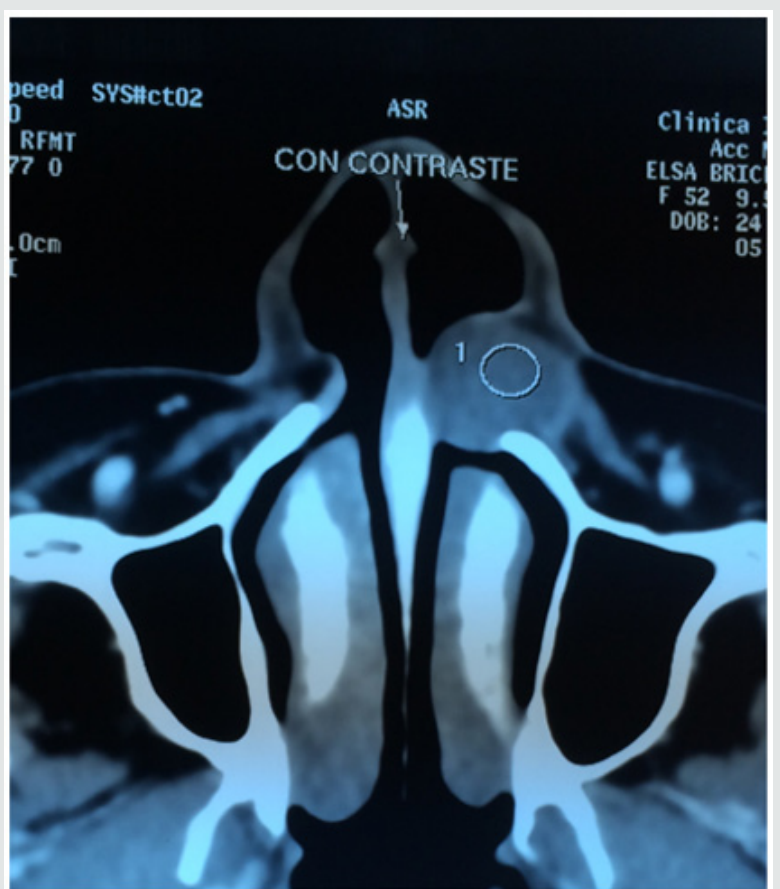

Figure 1(c): Computed tomography of left nasoalveolar cyst. Case 1.

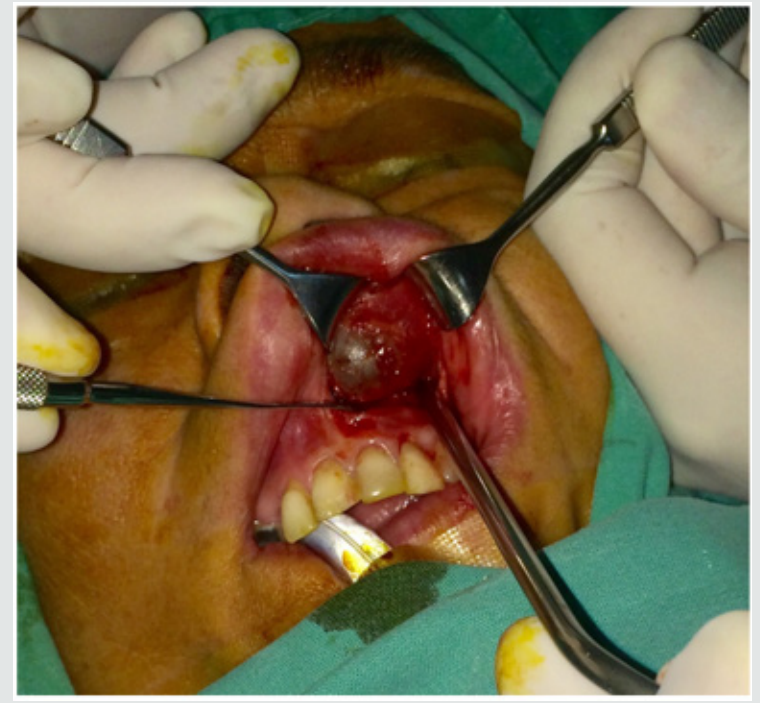

Figure 2: Intraoperative view of sublabial access to left nasolabial cyst in Case 1.

\section{Case Report 2}

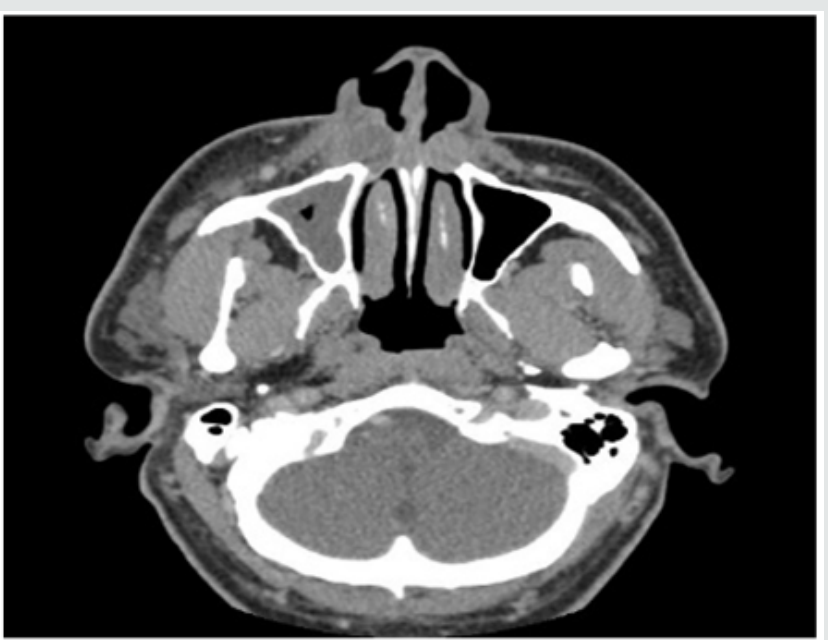

Figure 3: Computed tomography of bilateral nasoalveolar cysts. Case 2.

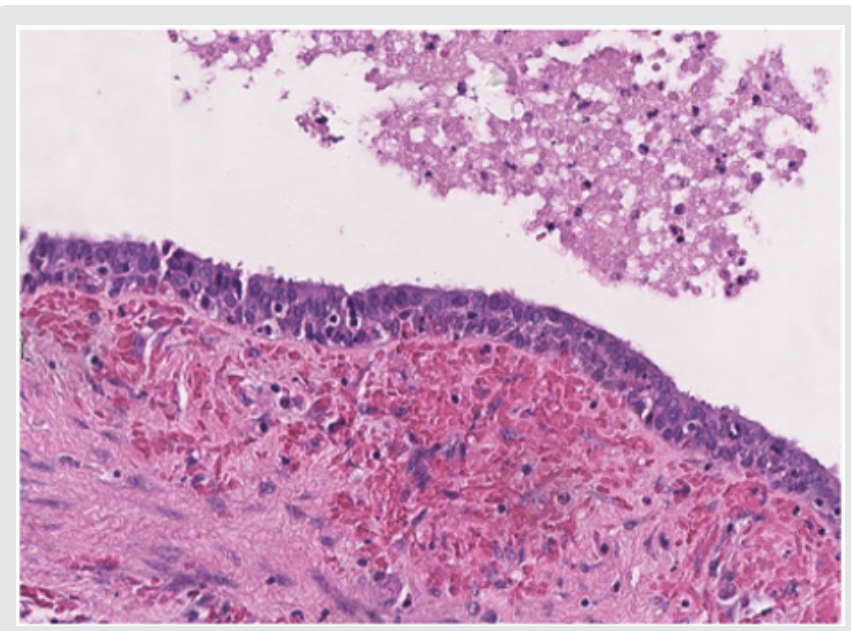

Figure 4: Pathology H-E study of Case 2 showing cyst wall with pseudostratified columnar cells. 
37-year-old male patient, with no prior medical record, developed a facial cellulitis with no initial response to oral antibiotics, requiring hospitalization and intravenous antibiotic treatment. Maxillary and orbital CT study showed bilateral nasoalveolar cysts, with signs of active infection in the right cyst (Figure 3). Antibiotic treatment was completed, and cyst resection was programmed a month later. An extensa sublabial approach was performed, and bilateral cysts were removed. Histological findings confirmed diagnosis (Figure 4). Patient did not have any other episode of facial cellulitis or cyst recurrence in a 14 month follow up

\section{Discussion}

Origin of nasolabial cysts is controversial. Most accepted theories are that cysts derive from remnants of the nasolacrimal duct or inclusion cysts of mesenchymal cells during the fusion of medial and lateral nasal prominences to the maxillary prominence during facial skeletal formation [2]. Histology usually present pseudostratified columnar epithelium. Su et al noticed with electronic microscopy that that cysts had a highly placated mucosa, of non-ciliated stratified columnar epithelium, differing from the ciliated columnar epithelium of the paranasal and nasal sinuses [7]. The treatment of nasolabial cysts consists on complete removal of the lesion, with the objective of prevention of infectious complications, histologic diagnosis, and aesthetic improvement. Fine needle aspiration can help in diagnosis, and relieve of symptoms, but recurrence is high [1]. Surgery is usually done by sublabial approach, creating a mucosal flap of gingiva to allow access to the pyriform aperture and to the cyst, which can be resected carefully to avoid rupture, especially to the floor of the nose mucosa, and complete excision is mandatory to avoid recurrence. After the intervention, the gingival mucosal flap is fixed in its original position with absorbable sutures. Potential complications are uncommon, including facial swelling, insensitive gingiva, teeth numbness, and surgical site infection.

Patients must use a toothbrush on the surgical site. Diet should be soft for the first week, then normal. Dental prostheses can be used immediately after surgery [1]. Lee et al published in 2009 a comparative study between surgical techniques, and strongly suggested endoscopic trans nasal marsupialization as a simpler, shorter and safer procedure, and in most cases, it could be done under local anesthesia [3]. In our two cases we used sublabial approach with general anesthesia because is the usual technique used in our practice, but we are interested to use endoscopic marsupialization in next cases. We did not have any complications or recurrences in follow up of both cases.

\section{Conclusion}

Nasolabial cysts are rare but must be considered in the differential diagnosis of floor of the nose deformities and facial swelling. Sublabial approach is the most common procedure, but probably be replaced by endoscopic marsupialization in the future.

\section{References}

1. Ordones AB, Neri L, Lopes Oliveira IH, Tepedino MS, Pinna Fde R, et al. (2013) Giant Nasolabial Cyst Treated Using Neumann Incision: Case Report. Int Arch Otorhinolaryngol 17: 421-423.

2. el Din K, el Hamd AA (1999) Nasolabial Cyst: a report of eight cases and a review of literature. J Laryngol Otol 113: 747-749.

3. Lee JY, Baek BY, Chang HS, Lee BD, Kim DW (2009) Comparison of conventional excision via a sublabial approach and transnasal marsupialization for the treatment of nasolabial cysts: a prospective randomized study. Clin Exp Otorhinolaryngol 37: 85-89.

4. Marcoviceanu MP, Metzger MC, Deppe H (2009) Report of rare bilateral nasolabial cysts. J Craniomaxillofac Surg 37: 83-86.

5. Choi JH, Cho JH, Kang HJ (2002) Nasolabial cyst: a retrospective analysis of 18 cases. Ear Nose Throat J 81: 94-96.

6. Aquilino RN, Bazzo VJ, Faria RJ (2008) Nasolabial cyst: presentation of a clinical case with CT and MR iamges. Braz J Otorhinolaryngol 74: 467471.

7. Su CY, Huang HT, Liu HY, Huang CC, Chien CY (2006) Scanning electron microscopic study of the nasolabial cyst: its clinical and embryological implications. Laryngoscope 116: 307:311.

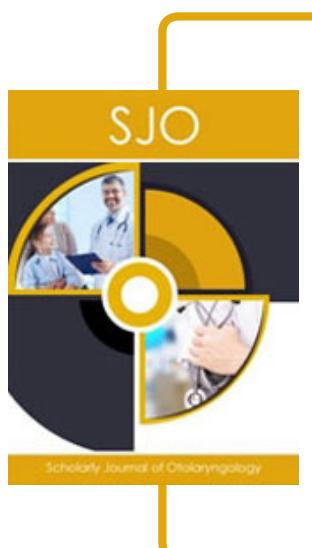

Scholarly Journal of Otolaryngology

\section{Assets of Publishing with us}

- Global archiving of articles

- Immediate, unrestricted online access

- Rigorous Peer Review Process

- Authors Retain Copyrights

- Unique DOI for all articles 